\section{Cavity cooling of a single atom}

P. Maunz, T. Puppe, I. Schuster, N. Syassen, P.W.H. Pinkse \& G. Rempe

Max-Planck-Institut für Quantenoptik,

Hans-Kopfermann-Str. 1, D-85748 Garching, Germany

All conventional methods to laser-cool atoms rely on repeated cycles of optical pumping and spontaneous emission of a photon by the atom. Spontaneous emission in a random direction is the dissipative mechanism required to remove entropy from the atom. However, alternative cooling methods have been proposed ${ }^{1,2}$ for a single atom strongly coupled to a high-finesse cavity; the role of spontaneous emission is replaced by the escape of a photon from the cavity. Application of such cooling schemes would improve the performance of atom cavity systems for quantum information processing ${ }^{3,4}$. Furthermore, as cavity cooling does not rely on spontaneous emission, it can be applied to systems that cannot be laser-cooled by conventional methods; these include molecules ${ }^{2}$ (which do not have a closed transition) and collective excitations of Bose condensates ${ }^{5}$, which are destroyed by randomly directed recoil kicks. Here we demonstrate cavity cooling of single rubidium atoms stored in an intracavity dipole trap. The cooling mechanism results in extended storage times and improved localization of atoms. We estimate that the observed cooling rate is at least five times larger than that produced by free-space cooling methods, for comparable excitation of the atom.

The basic idea behind cavity cooling can be understood from a simple classical picture based on the notion of a refractive index. Consider a standing-wave optical cavity resonantly excited by a weak probe laser blue detuned from the atomic resonance. For strong atom-cavity coupling, even one atom can significantly influence the optical path length between the cavity mirrors. Consequently, the intracavity intensity is strongly affected by the atom ${ }^{6-8}$. For example, at a node of the standing wave the atom is not coupled to the cavity, thus the intracavity intensity is large. An atom at an antinode, in contrast, shifts the cavity to a higher frequency because the atom's refractive index is smaller than unity above its resonance. This tunes the cavity out of resonance from the probe laser and leads to a small intracavity intensity. However, in a high-finesse cavity the intensity cannot drop instantaneously when the atom moves away from a node. Instead, the blue-shift of the cavity frequency leads to an increase of the energy stored in the field. The photons finally escaping from the cavity are therefore blue-shifted from the photons of the probe laser. This occurs at the expense of the atom's kinetic energy. The reverse effect, namely the acceleration of an atom approaching an antinode, is much smaller as here the cavity is initially out of resonance with the probe laser and consequently the intracavity intensity is small.
Note that the cooling process does not require atomic excitation. Indeed, the atomic excitation is low at all times as the atom is not coupled to the light at a node while the intracavity intensity is very low for an atom near an antinode. It follows that the lowest attainable temperature is not limited by the atomic linewidth as for free-space Doppler cooling but by the linewidth of the cavity, which can be much smaller. Therefore temperatures below the Doppler limit can be reached ${ }^{9}$. An upper limit on the velocity of the atom to be cooled is given by the requirement that the atom must not move farther than about one-quarter of a wavelength during the lifetime of a photon in the cavity. In our experiment, this corresponds to a velocity of about $3 \mathrm{~m} / \mathrm{s}$. We emphasise that cavity cooling is applied to a single two-level atom and differs from the mechanical effects observed for an atomic ensemble ${ }^{10-12}$. A description of cavity cooling in terms of dressed-states pictures of the strongly coupled atom-cavity system can be found elsewhere ${ }^{9}$.

A treatment of cavity cooling combined with trapping by means of an auxiliary far-red detuned dipole laser is quantitatively different. It can be achieved by including the dynamic Stark shift of the atomic ground and excited states into the Hamiltonian. The dynamic Stark shift renders the atomic resonance frequency position dependent, making it larger for an atom at an antinode. This effect even enhances the cooling force by effectively increasing the refractive index variations for a moving atom. The combined system can be investigated numerically ${ }^{13}$. Moreover, in the limit of low atomic excitation analytic expressions for all relevant forces including the cooling force can be derived, thereby extending previous calculations ${ }^{1,9}$. The obtained expressions are lengthy but valuable for parameter optimisation and straightforward trajectory calculation.

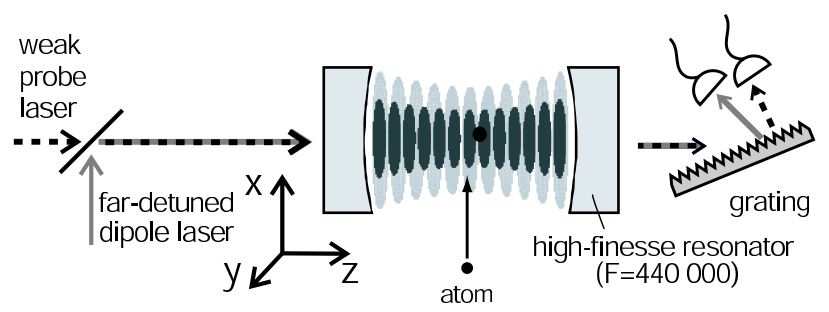

Figure 1: Experimental set-up. The high-finesse cavity $(\mathcal{F}=$ $\left.4.4 \times 10^{5}\right)$ is excited by a weak near-resonant probe field and a strong far-red detuned dipole field. ${ }^{85} \mathrm{Rb}$ atoms are injected from below. Behind the cavity, the two light fields are separated by a grating. The probe light is further passed through a narrow-band interference filter before being directed onto a single-photon counting module. For this set-up, a quantum efficiency of $32 \%$ is achieved for the detection of probe light transmitted through the cavity while the dipole light is attenuated by more than $70 \mathrm{~dB}$. The dipole light is also used to stabilize the cavity length with a radio frequency sideband technique. It is generated by a grating- and current-stabilized diode laser with a linewidth of $20 \mathrm{kHz}$ r.m.s. 
In our experiment, see Fig. 11 the cavity has a finesse $\mathcal{F}=4.4 \times 10^{5}$ and a length $l=120 \mu \mathrm{m}$. The cavity field has a decay rate $\kappa / 2 \pi=1.4 \mathrm{MHz}$. The wavelength difference between neighbouring longitudinal $\mathrm{TEM}_{00}$ modes is about $2.5 \mathrm{~nm}$. Single laser-cooled ${ }^{85} \mathrm{Rb}$ atoms are injected into the cavity ${ }^{7}$ with a velocity smaller than $10 \mathrm{~cm} / \mathrm{s}$. The single-photon coupling constant is $g / 2 \pi=16 \mathrm{MHz}$ for the $5^{2} S_{1 / 2} F=3 \leftrightarrow 5^{2} P_{3 / 2} F=4$ transition with dipole decay rate $\gamma / 2 \pi=3 \mathrm{MHz}$. A weak, near-resonant probe laser at $780.2 \mathrm{~nm}$ is used to observe and cool the atom. A strong, far-detuned dipole laser at $785.3 \mathrm{~nm}$ serves to trap the atom. The detunings of the probe laser with respect to the cavity, $\Delta_{c}=0$, and the atom, $\Delta_{a} / 2 \pi=35 \mathrm{MHz}$, are chosen to compromise between ideal detunings for detection and good cooling conditions while maintaining a very low excitation of the atom at any moment of the experiment. In fact, the presence of an atom at an antinode reduces the transmission of the probe light by a factor of 100 , allowing its detection and manipulation with a high signal-to-noise ratio and a high bandwidth $^{14-17}$.

Experiments are performed only with atoms located in the central region of the cavity, where the nodes and antinodes of the probe and dipole fields coincide. This is accomplished by turning on the dipole laser before injecting the atom into the cavity. In this case, a $400 \mu \mathrm{K}$ deep dipole potential guides the arriving atom into the high-intensity region. Only if this region is also a region of strong coupling, the presence of an atom manifests itself as a pronounced dip in the cavity transmission as monitored with the probe light. Atoms entering the cavity at an axial position where the two standing waves are out-of-phase are confined to nodes of the probe field and, hence, are invisible to the probe laser. If the transmission drops below $9 \%$, the intensity of the dipole light is increased to generate a trap depth of about $1.5 \mathrm{mK}$. This compensates for the radial kinetic energy of the atom and enables to catch the atom in an otherwise conservative potential. More than $95 \%$ of the detected atoms are captured in the dipole trap.

The average storage time of the atom in the dark intracavity trap is measured by turning off the probe light for an adjustable time interval, $\Delta t$, after the atom is captured. As a function of the dark time, $\Delta t$, the fraction of atoms still trapped drops exponentially with a decay constant of $18 \mathrm{~ms}$, defining the storage time of the atoms in the trap. The theoretical limits imposed by light scattering $(85 \mathrm{~s})$ and genuine cavity QED dipole fluctuations $(200 \mathrm{~ms})^{1}$ cannot explain this rather short time ${ }^{18,19}$. Instead the observed loss of the atom is attributed to parametric heating due to fluctuations of the intracavity intensity mainly caused by frequency fluctuations of the dipole laser. This technical noise critically depends on the tuning of the laser stabilization. Indeed, the storage time in the dark dipole trap could be increased from $18 \mathrm{~ms}$ to $31 \mathrm{~ms}$ by improving the frequency stability of the dipole laser. As this stability and other sources of noise are hard to control, a concurrent measurement of the storage time of the dark dipole trap serves as a reference in each of the following experiments. Note that the axial trap frequency is about 100 times higher than the radial trap frequency. As parametric heating is proportional to the square of the trap frequency ${ }^{20}$ the atom is mainly heated in axial direction. Since axial and radial motion are only weakly coupled, the heated atom usually escapes the antinode of the standing wave dipole trap along the axis, thereby hitting one of the mirrors. This conjecture is supported by numerical simulations of the experiment, as further discussed below.

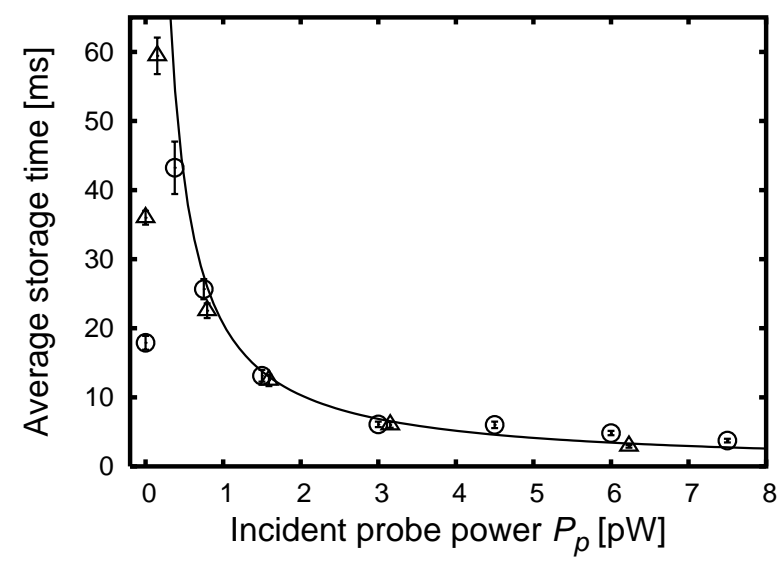

Figure 2: Storage time. The storage time of a trapped atom as a function of probe power. Two sets of data taken before (circles) and after (triangles) improving the laser stabilization are displayed. The corresponding storage times in the dark trap are $18 \mathrm{~ms}$ and $36 \mathrm{~ms}$, respectively. For an incident probe power exceeding about $0.5 \mathrm{pW}$ the storage time is limited by radial escape due to spontaneous emission. In this range the measured storage time (in ms) can be approximated by $20 / P_{p}$ (where $P_{p}$ is in units of $\mathrm{pW}$; solid line). The storage time of $18 \mathrm{~ms}$ obtained for the dark trap increases by more than a factor of two by applying $0.37 \mathrm{pW}$ probe light. After the stabilization of the dipole laser was improved, $P_{p}=0.11 \mathrm{pW}$ increased the storage time from $36 \mathrm{~ms}$ to $60 \mathrm{~ms}$. The mentioned probe powers correspond to an average intracavity photon number of 0.005 and 0.0015 for $0.37 \mathrm{pW}$ and $0.11 \mathrm{pW}$, respectively.

To demonstrate that cavity cooling can be used to compensate for the axial heating of the dipole trap, the probe beam is not switched off completely after capturing an atom. Fig. 2 shows the storage time as a function of the incident probe power, $P_{p}$. For high probe power the storage time is reduced as compared to the dark trap. However, the storage time increases with decreasing power. This effect is attributed to the reduction of spontaneous emission which heats the atom in all directions and which cannot be compensated radially since cavity cooling acts mainly axially. As the atomic transition is still far from being saturated, the radial heating is proportional to the probe power even for the highest considered level of $P_{p}=7.5 \mathrm{pW}$. Hence, as long as the probe power is large enough to compensate for axial heating, the storage time $\tau$ 
is limited by radial loss and $\tau \propto P_{p}^{-1}$ (solid line). Consequently, the atom is expected to leave the cavity axially for near-zero probe power, while in case of higher power radial losses should dominate. This is confirmed by a Monte Carlo simulation of a point-like atom moving in the trap under the influence of the forces and momentum diffusion calculated analytically, while parametric heating from the dipole trap is implemented by a randomly changing potential depth. The storage times evaluated from the simulation agree well with the experiment. Moreover it can be concluded that for probe powers below $0.1 \mathrm{pW}$ more than $90 \%$ of the atoms leave the cavity by hitting a mirror, while for higher probe powers $90 \%$ of the atoms leave radially.

We emphasise that, in contrast to Doppler cooling, cavity cooling extends the storage time for a probe field which is blue detuned from the atom. If the detuning is changed from blue to red by adjusting the atom-cavity detuning while keeping the dipole power constant and the probe laser resonant with the cavity, the average storage time decreases and drops below the storage time of the dark trap. This clearly demonstrates that the extension of the storage time cannot be attributed to Doppler cooling.

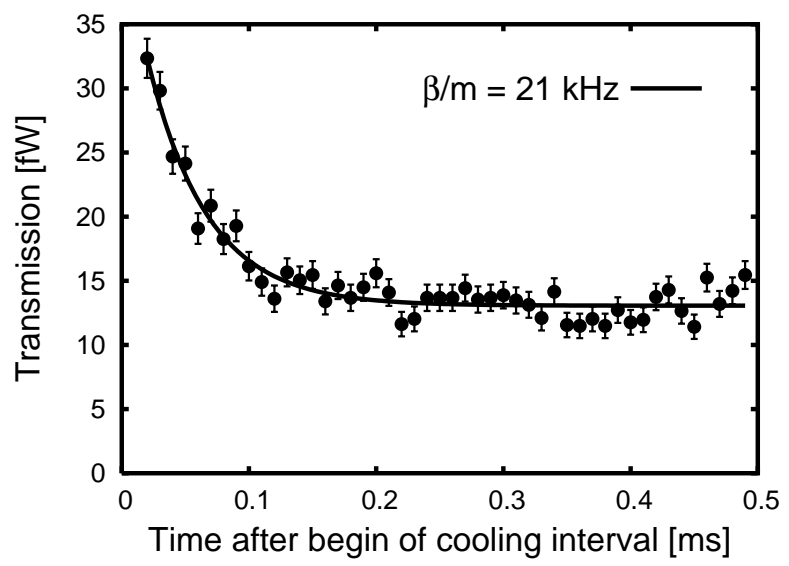

Figure 3: Cooling-induced localization. Average transmission during cooling intervals of length $0.5 \mathrm{~ms}$ after heating the atom for $0.1 \mathrm{~ms}$. An incident power of $P_{p}=2.25 \mathrm{pW}$ is chosen for a good signal to noise ratio. Without an atom, the cavity transmission on resonance is $300 \mathrm{fW}$. The atoms are cooled during the first $0.1 \mathrm{~ms}$. This leads to a stronger coupling to the cavity mode and, hence, to a smaller transmission. A cooling rate of $\beta / m=21 \mathrm{kHz}$ is estimated from an exponential fit. Radial heating occurs on a much longer timescale and is not visible here.

We now demonstrate cooling by directly observing the reduction of the kinetic energy of the atom. The experiment employs alternating heating and cooling intervals at a constant probe power of $P_{p}=2.25 \mathrm{pW}$. Axial heating is achieved by deliberately tuning the probe laser for a time interval of $100 \mu \mathrm{s}$ to a frequency $9 \mathrm{MHz}$ above the cavity resonance $\left(\Delta_{c} / 2 \pi=9 \mathrm{MHz}\right)$, where strong heating of the atom is ex- pected from the theoretical analysis. In the following $500 \mu \mathrm{s}$ long cooling interval the probe laser is switched back to the cavity resonance $\left(\Delta_{c}=0\right)$. Fig. 3 shows the cavity transmission averaged over many cooling intervals, with an atom present in the cavity. The transmission drops by more than a factor of two during the first $100 \mu \mathrm{s}$. This drop is a clear signature for the increasing atom-cavity coupling, and hence, a better localization of the atom at the antinode.

The exponential change of the transmission observed for short times allows to obtain an estimate of the mean cooling rate, $\beta / m=21 \mathrm{kHz}$, where $\beta$ is the friction coefficient and $m$ the atomic mass. This result is in good agreement with the Monte Carlo simulations, which show an exponential relaxation with a timescale of about $50 \mu$ s for the increase of the atomic localization as well as for the decrease of the transmitted power. To compare this rate of cavity cooling with free-space cooling rates of a two-level atom for a given rate of spontaneous emission events, knowledge about the atomic excitation is required. Here, an upper limit can be obtained by attributing the storage time of $9 \mathrm{~ms}$ (measured for a probe power of $2.25 \mathrm{pW}$; Fig. 2. solely to radial heating from spontaneous emission. To leave the trap, an atom must have gained about $1 \mathrm{mK}$ of kinetic energy. This limits the atomic excitation to below $2.5 \%$. At this excitation, free-space Sisyphus cooling ${ }^{21,22}$ of a two-level atom in a blue-detuned standing wave would achieve $\beta_{\mathrm{S}} / m=4 \mathrm{kHz}$ while Doppler cooling would have $\beta_{\mathrm{D}} / m=1.5 \mathrm{kHz}$, both for optimal detuning. Thus introducing the cavity increases the cooling rate by at least a factor of 5 for constant atomic excitation.

Cooling down an atom in the trap can also be demonstrated without the additional heating. For this purpose the atom is repeatedly left in the dark dipole trap by switching off the probe light during $2 \mathrm{~ms}$ long time intervals. These intervals are short compared to the average trapping time in the dark trap of $31 \mathrm{~ms}$, obtained after improving the frequency stability of the dipole laser. In each of the dark intervals the atom experiences parametric heating. Between the dark intervals, $100 \mu$ s long cooling intervals are applied using a probe power $P_{p}=1.5 \mathrm{pW}$ on resonance with the cavity $\left(\Delta_{c}=0\right)$. The transmission of the probe light is also used to determine whether the atom is still present. The average time an atom is stored in the trap under these conditions can be calculated by adding up all the $2 \mathrm{~ms}$ long dark intervals after which the atom is still found in the trap, but omitting the cooling intervals. The result is shown in Fig. 4 although the short cooling intervals have a duty cycle of only $5 \%$ they increase the average trapping time by more than $50 \%$. Obviously, heating the atom out of the trap requires more time in the presence of cooling. Therefore the kinetic energy of the atom was reduced during the cooling interval.

In conclusion, strong coupling of a two-level atom to the cavity field was used to cool single atoms stored in an intracavity dipole trap. The storage time in the trap has been 


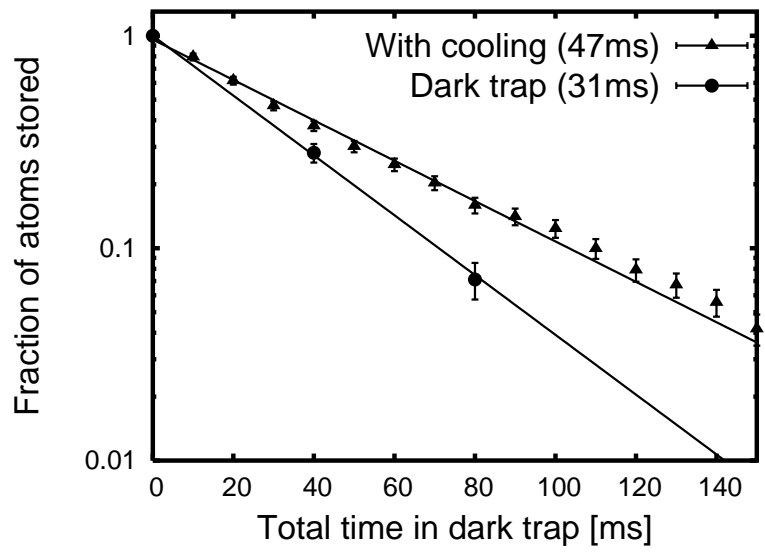

Figure 4: Cavity cooling. The fraction of atoms stored in the trap as a function of time after the dipole trap is switched on. The dark dipole trap has an average storage time of $31 \mathrm{~ms}$ (circles), as obtained from an exponential fit. If $100 \mu$ s long cooling intervals are applied every $2 \mathrm{~ms}$, the average storage time without counting the cooling intervals is extended to $47 \mathrm{~ms}$ (triangles). The storage time in the dark trap varies slightly from day to day and is therefore measured concurrently. As in several detailed measurements (not shown) the fraction of atoms found in the dark trap as a function of time was found to be very well described by an exponential decay, three data points (including the one at zero trapping time) are sufficient to obtain the storage time in the dark trap.

increased by a factor of two by exploiting the cooling force caused by a near-resonant cavity field with an average photon number of only 0.005 . In contrast to free-space laser cooling techniques, this cooling force acts mainly by exciting the cavity part of the coupled atom-cavity system. Thus strong cooling forces can be achieved while keeping the atomic excitation low. An estimate of the strength of the cooling force has shown to exceed the force expected for free-space Sisyphus cooling and Doppler cooling at comparable atomic excitation by at least a factor of 5 and 14, respectively. Avoiding excitation could serve as a basis for cooling of molecules or collective excitations of a Bose condensate ${ }^{5}$. Another application might be to cool the motion of an atom with a stored quantum $\mathrm{bit}^{23}$. If the two states forming the qubit have identical coupling to the cavity, the new cooling scheme would not disturb the superposition state. This advantage is not shared by any other cooling method.

\section{References}

1. P. Horak, G. Hechenblaikner, K.M. Gheri, H. Stecher, and H. Ritsch, Cavity-Induced Atom Cooling in the Strong Coupling Regime. Phys. Rev. Lett. 79, 4974-4977 (1997).

2. V. Vuletić and S. Chu, Laser Cooling of Atoms, Ions, or Molecules by Coherent Scattering. Phys. Rev. Lett. 84, 3787-3790 (2000).
3. A. Kuhn, M. Hennrich, and G. Rempe, Deterministic Single-Photon Source for Distributed Quantum Networking. Phys. Rev. Lett. 89, 067901 (2002).

4. for a review, see e.g., C. Monroe, Quantum information processing with atoms and photons. Nature 416, 238-246 (2002).

5. P. Horak, and H. Ritsch, Dissipative dynamics of Bose condensates in optical cavities. Phys. Rev. A 63, 023603 (2001).

6. H. Mabuchi, Q.A. Turchette, M.S. Chapman, and H.J. Kimble, Real-time detection of individual atoms falling through a high-finesse optical cavity. Opt. Lett. 21, 1393-1395 (1996).

7. P. Münstermann, T. Fischer, P.W.H. Pinkse, and G. Rempe, Single slow atoms from an atomic fountain observed in a high-finesse optical cavity. Opt. Commun. 159, 63-67 (1999).

8. J.A. Sauer, K.M. Fortier, M.S. Chang, C.D. Hamley, and M.S. Chapman, Cavity QED with optically transported atoms. http://arXiv.org/quant-ph/0309052 (2003).

9. G. Hechenblaikner, M. Gangl, P. Horak, and H. Ritsch, Cooling an atom in a weakly driven high-Q cavity. Phys. Rev. A 58, 3030-3042 (1998).

10. H.W. Chan, A.T. Black, and V. Vuletić, Observation of Collective-Emission-Induced Cooling of Atoms in an Optical Cavity. Phys. Rev. Lett. 90, 063003 (2003).

11. B. Nagorny, Th. Elsässer, and A. Hemmerich, Collective Atomic Motion in an Optical Lattice Formed Inside a High Finesse Cavity. Phys. Rev. Lett. 91, 153003 (2003).

12. D. Kruse, C. von Cube, C. Zimmermann, and Ph.W. Courteille, Observation of Lasing Mediated by Collective Atomic Recoil. http://arXiv.org/quant-ph/0305033 (2003).

13. S.J. van Enk, J. McKeever, H.J. Kimble, and J. Ye, Cooling of a single atom in an optical trap inside a resonator. Phys. Rev. A 64, 013407 (2001)

14. C.J. Hood, T.W. Lynn, A.C. Doherty, A.S. Parkins, and H.J. Kimble, The Atom-Cavity Microscope: Single Atoms Bound in Orbit by Single Photons. Science 287, 1447-1453 (2000).

15. P.W.H. Pinkse, T. Fischer, P. Maunz, and G. Rempe, Trapping an atom with single photons. Nature 404, 365-368 (2000).

16. P. Münstermann, T. Fischer, P. Maunz, P.W.H. Pinkse, and G. Rempe, Dynamics of Single-Atom Motion Observed in a High-Finesse Cavity. Phys. Rev. Lett. 82, 3791-3794 (1999).

17. T. Fischer, P. Maunz, P.W.H. Pinkse, T. Puppe, and G. Rempe, Feedback on the motion of a single atom in an optical cavity. Phys. Rev. Lett. 88, 163002 (2002)

18. Y. Ye, D.W. Vernooy, and H.J. Kimble, Trapping of Single Atoms in Cavity QED. Phys. Rev. Lett. 83, 4987-4990 (1999).

19. J. McKeever, et al. State-Insensitive Cooling and Trapping of Single Atoms in an Optical Cavity. Phys. Rev. Lett. 90, 133602 (2003).

20. T.A. Savard, K.M. O'Hara, and J.E. Thomas, Laser-noise-induced heating in far-off resonance optical traps. Phys. Rev. A 56, R1095-R1098 (1997).

21. A. Aspect, J. Dalibard, A. Heidmann, C. Salomon, and C. CohenTannoudji, Cooling Atoms with Stimulated Emission. Phys. Rev. Lett. 57, 1688-1691 (1986)

22. J. Dalibard, and C. Cohen-Tannoudji, Dressed-atom approach to atomic motion in laser light: the dipole force revisited. J. Opt. Soc. Am. B 2, $1707-$ 1720 (1985)

23. A. Griessner, D. Jaksch, and P. Zoller, Cavity Assisted Nondestructive Laser Cooling of Atomic Qubits. http://arXiv.org/quant-ph/0311054 (2003).

Correspondence and requests for materials should be addressed to G. R. (e-mail: gerhard.rempe@mpq.mpg.de). 